\title{
Pigeons' (Columba livia) Encoding of Geometric and Featural Properties of a Spatial Environment
}

\author{
Debbie M. Kelly, Marcia L. Spetch, and C. Donald Heth \\ University of Alberta
}

\begin{abstract}
Pigeons (Columba livia) searched for hidden food in a rectangular environment constructed to eliminate external orientation cues. A feature group was initially trained with distinct features in each comer. A geometric group was initially trained with no featural information. Tests revealed that both groups encoded the geometry of the apparatus. The geometric group was then retrained with features, and a series of tests was administered to both groups. Transformation tests revealed that the groups differed in reliance on features versus geometry. Pigeons in the feature group followed the positive feature even when it was placed in a geometrically incorrect comer, whereas pigeons in the geometric group showed shared control by features and geometry. Both groups were able to use features in comers distant to the goal to orient themselves, and both groups relied more on the color than on the shape of the features.
\end{abstract}

Survival within an environment frequently requires efficient processing of spatial information. Spatial abilities underlie activities that are critical for the individual (e.g., establishment of lodging, avoidance of predation, and attainment of nourishment) and for a species (e.g., migratory behavior or reproduction); these activities may involve a variety of mechanisms. Navigation, for example, may be achieved through inertial guidance, orientation to a beacon, piloting by use of landmarks, or developing a spatial representation of the environment (Gallistel, 1990).

Questions concerning which aspects of an environment are encoded and used in navigation have been addressed in recent research (for reviews, see Cheng \& Spetch, 1998; Gallistel, 1990; and Poucet, 1993). Many studies have shown that animals can encode and use multiple sources of information to locate a goal (e.g., Spetch \& Edwards, 1988) and that the primacy of control by different sources of information may differ according to context (e.g., Strasser \& Bingman, 1996) or species (e.g., Brodbeck, 1994). One particularly interesting set of results has emerged from studies that have controlled and manipulated the information available for encoding by restricting access to navigational cues in an enclosed environment and by disrupting other positional cues through disorientation techniques (Cheng, 1986; Hermer \& Spelke, 1994, 1996; Vallortigara, Zanforlin,

Debbie M. Kelly, Marcia L. Spetch, and C. Donald Heth, Department of Psychology, University of Alberta, Edmonton, Alberta, Canada.

This research was supported by a Natural Sciences and Engineering Research Council of Canada research grant. We thank D. Wahlsten for statistical advice and L. Price for assistance with the graphics.

Correspondence concerning this article should be addressed to Marcia L. Spetch, Department of Psychology, University of Alberta, Edmonton, Alberta, Canada T6G 2E9. Electronic mail may be sent to mspetch@psych.ualberta.ca.
\& Pasti, 1990). These studies have compared control by navigational cues that depend on the geometry of the environment with control by cues provided by specific perceptual features in the environment. Cheng (1986) and Cheng and Gallistel (1984) demonstrated that rats rely primarily on the geometry of the environment to determine a target position. For example, Cheng (1986) trained rats in a reference memory task to find food that was located in one corner of a rectangular environment containing distinctly different features in each corner. Control by geometry was indicated by the occurrence of systematic rotational errors, in which rats were more likely to choose the corner that was geometrically equivalent to the correct corner (but contained the wrong feature) than the corners that were geometrically incorrect. These errors to the geometrically correct corner indicate that the rats had encoded the metric properties of the environment. Although the rats did eventually learn to use the features to distinguish between the two geometrically correct corners, tests with various transformations of the environment revealed that geometry rather than the featural cues exerted primary control over the rats' search behavior. Specifically, the rats did not follow the correct feature when it was moved to a geometrically incorrect corner. Additional evidence for the primacy of geometric cues in rats was provided by Margules and Gallistel (1988).

Vallortigara et al. (1990) showed that chicks trained in a similar environment also demonstrated systematic rotational errors when the training features were removed or when novel uninformative features were provided. However, unlike the rats, responses by the chicks on transformation tests showed a strong reliance on the feature associated with the positive comer even when it was placed in a geometrically incorrect position. Therefore, the chicks encoded both the featural and geometric information, but they showed a preference for the featural information. They used geometric information only when features were unavailable or uninformative. 
In addition to these apparent phylogenetic differences, experiential or developmental factors might also affect the use of spatial information. Hermer and Spelke (1994) presented human subjects with a task similar to that used by Cheng (1986) and Vallortigara et al. (1990). The researchers hid an object in one corner of either a uniformly painted rectangular room or a room with one wall painted blue. University students and young children (18 to 24 months) were disoriented after witnessing the experimenter hide the object. The subjects were then instructed to locate the object in the room. Although the university students were able to identify which corner contained the hidden object when provided with one blue wall, the young children confused the two geometrically equivalent corners. Even after distinct objects were placed in the corners and the children's attention was drawn to the placement of the object, the children still did not use these features to reorient themselves.

Our experiment examined questions similar to those addressed by Cheng (1986) and Vallortigara et al. (1990), both of whose results suggest that different species may use different types of information to locate objects in space. Another avian species might, like the chicks of the Vallortigara et al. study, rely on featural more than geometric information. Hermer and Spelke's (1994) results suggest that age-related factors, such as the greater navigational experience of adults, also affect the use of featural information. In our experiment, we tested pigeons to determine their reliance on featural information under different types of initial experience with geometric or featural information.

Thus, our experiment differed from previous studies in a number of respects. First, the pigeons in our experiment were divided into two groups depending on the type of initial training they were to receive. Manipulating the type of training allowed us to investigate potential training effects. We hypothesized that control by geometry would be stonger in subjects initially trained without features than in subjects initially trained with both features and geometry. Second, the featurally trained subjects were further subdivided according to the type of featural information they were to receive. For half of these subjects, the features consisted of three-dimensional landmarks; for the other half of the subjects, the features were flat panels affixed to the corners of the apparatus. We expected that the landmark objects would be more salient because Chappell and Guilford (1997) found that pigeons were unable to locate a target using two-dimensional panels on the walls of an octagonal enclosure but were able to locate it using a threedimensional landmark. Third, our experiment included a larger number of transformation tests than in previous studies, thereby providing a more comprehensive investigation of responses to tests that pitted geometry against featural information. Fourth, we included transfer tests to identify which property or properties of the features (shape, color, or three dimensionality) were encoded. Finally, we included a test in which the apparatus was altered physically to examine the nature of the geometric information encoded.

\section{Method}

\section{Subjects}

Eight adult silver king pigeons (Columba livia) participated as subjects in this experiment. The pigeons had previous experience in an unrelated time discrimination task conducted in a standard operant chamber. The pigeons were maintained at $85 \%$ of their free-feeding body weight throughout the duration of this experiment. We provided maple peas as reinforcement during the experiment and supplemental feedings of KayTee pigeon pellets as required to maintain the pigeons at their individual experimental weights. The pigeons were individually housed and exposed to a 12-hr light-dark schedule with light onset at 0600 .

\section{Apparatus}

The experimental apparatus was a uniformly white, rectangular enclosure assembled to control for cue availability (similar to that used by Cheng, 1986). The walls were constructed from opaque plastic and 5-cm thick Styrofoam. The construction of the apparatus was such that no discernable differences could be detected between the two short walls or between the two long walls. Specific measurements of the outside dimensions of the entire apparatus are provided in Figure 1. The inside dimensions of the enclosure were $90 \times 190 \mathrm{~cm}$. The floor of the enclosure was covered with approximately $5 \mathrm{~cm}$ of aspen chip bedding. A video camera mounted above the apparatus monitored the inside of the experimental apparatus and recorded responses. The video camera was hidden behind a $30-\mathrm{cm}$ suspended ceiling so that only the lens of the camera remained visible. Four small Velcro squares were affixed to the flooring, $24 \mathrm{~cm}$ from each corner. Four identical round tin containers (8.5-cm diameter and $3.5-\mathrm{cm}$ height) containing $2 \mathrm{~cm}$ of pigeon grit were later positioned on the Velcro squares. White noise was played through four speakers to mask any exterior noise cues, with one speaker outside each corner of the apparatus. The positions of individual speakers were intermittently exchanged at random.

\section{Features}

The stimuli used as features in this experiment can be divided into two categories: landmarks and panels. For each subject, the features were either all landmarks or all panels. Landmarks and panels were placed flush into each comer so that they physically touched both walls. The landmarks were three-dimensional objects of unique color and shape. The panels approximated the landmarks in color and shape but were constructed from 3-mm cardboard, thus providing a two-dimensional feature array. Figure 1 provides the dimensions of the various landmarks and panels used during training and testing.

\section{Shaping Procedures}

The pigeons were initially trained to eat out of a tin container while they were in their home cages. As soon as a pigeon had eaten out of the container without hesitation, a piece of paper towel was placed over the container and secured with a rubber band so that the pigeon was required to peck through the paper towel cover to obtain the food. Shaping in the experimental apparatus began once a pigeon readily pecked through the paper towel covering.

The 8 pigeons were divided into two groups (of 4 subjects each) according to the type of training they were to receive initially. One 


\section{APPARATUS}

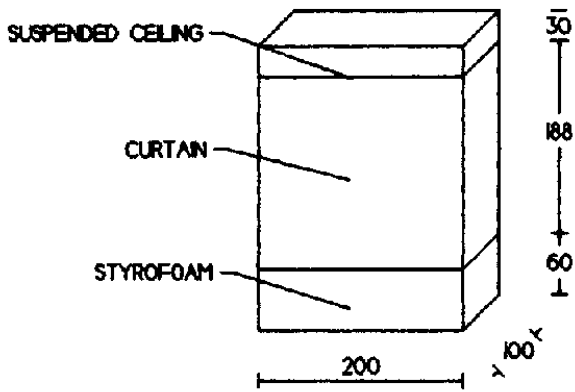

TRAINING LANDMARKS

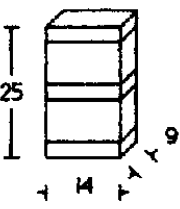

A

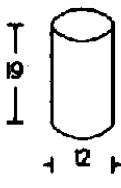

$B$

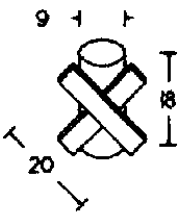

C

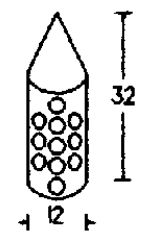

TRAINING PANELS

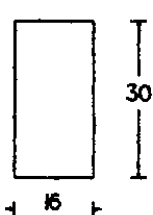

C

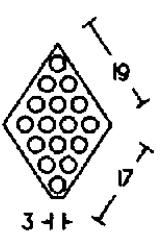

D

\section{TEST LANDMARKS}

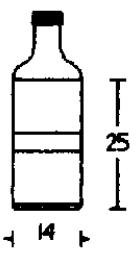

I

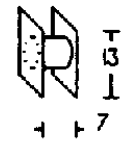

CT

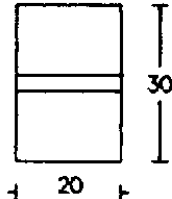

I<smiles>C1CCCCC1</smiles>

CT

Figure 1. The apparatus, landmarks, and panels used in the study. The top illustration shows the dimensions of the experimental apparatus. The next three rows of illustrations show the dimensions of the landmarks and panels used for training and testing. All dimensions are reported in centimeters. The training landmarks and panels labeled $A$ were pink with black stripes, those labeled $B$ were green, those labeled $C$ were blue, and those labeled $D$ were red with orange circles. The test landmark and panel labeled $I$ were used for the Identical Features Tests and were yellow with a brown stripe. The test landmark and panel labeled $C T$ were the novel shapes used for the color transfer tests and were presented in the colors of the training landmarks and panels.

group of subjects received training in the apparatus without the presence of any distinct featural information (the geometric group). The other group of subjects (the feature group) was trained with relevant and distinctly different featural information provided at each of the four comers. Each of the birds in the geometric group was assigned to a different "positive" comer. On reinforced trials, food was available only at this positive comer, and containers at the other three corners never had food. The birds in the feature group were also each assigned to a different positive corner. However, the birds in the feature group were further subdivided into two groups ( 2 subjects in each) depending on the type of featural information (landmarks or panels) provided at the four corners.

The pigeons were given one experimental session per day; it consisted of 10 trials. The pigeons were transported to and from the 
experimental room in an opaque plastic jug. Before each trial, the pigeon was placed in an opaque holding cage on a swiveling chair (the placement of the chair relative to the experimental apparatus was randomized on each trial) and rotated for $1 \mathrm{~min}$ at approximately 12 revolutions per minute to eliminate any use of inertial cues. The subject was then placed into the apparatus according to a schedule of randomly chosen entry positions (one on each wall). The subject was given $10 \mathrm{~min}$ to make a choice. The first peck that broke the paper towel covering a container was considered a choice. A subject was permitted to make two choices per trial, after which the lights in the room were dimmed, and the subject was removed from the apparatus. The point of exit from the apparatus was determined by a prearranged schedule of randomly chosen exit positions. Entry and exit positions were located at the midpoint of each of the four walls. After completion of a trial, the subject was placed into the holding cage while the bedding in the apparatus was sifted and any extraneous debris was removed, and the apparatus was prepared for a new trial. The subject was then rotated and a new trial was begun.

The pigeons' training was divided into five shaping phases designed to train persistent searching behavior under conditions of degraded information. The first shaping phase allowed the pigeon to adjust to the novel surroundings. One tin container was placed at the bird's positive comer. The container was not covered, and four maple peas were placed on the grit. A subject was allotted $10 \mathrm{~min}$ to eat from the container. If a subject ate from the container, it was given 1 additional min, after which the lights were dimmed, and the subject was removed from the apparatus. Failure to eat from the container after $10 \mathrm{~min}$ resulted in termination of the trial and the start of a new trial immediately thereafter. Successful completion of Shaping Phase 1 required that a pigeon eat the maple peas from the container within $5 \mathrm{~min}$ on each trial and complete all 10 trials. Two pigeons failed to eat when placed in the experimental chamber; after 7 days, they were dropped from the study and 2 new birds with identical experimental histories were added.

The second shaping phase was identical to the first with the exception that an uncovered container was placed at each of the four corners; only the container in the positive corner contained food. The criteria for Shaping Phase 2 were the same as for Shaping Phase 1.

The third shaping phase was similar to Shaping Phase 2, except that each of the containers was covered with a paper towel. Successful completion of Shaping Phase 3 included the criteria used in previous shaping sessions plus an accuracy criterion. For feature training, $80 \%$ or more of the first choices were required to be directed to the container at the positive corner. For the geometric group, there were no cues that would allow the pigeon to distinguish the positive corner from the geometrically equivalent comer. Therefore, we required that $80 \%$ of first choices be directed to either of the two geometrically equivalent positive corners.

The fourth and fifth shaping phases were instituted to prepare the birds for the density of reinforcement that they would experience during testing sessions. The schedule of reinforcement was set at $70 \%$ in Shaping Phase 4 and 50\% in Shaping Phase 5. The subjects were allowed $10 \mathrm{~min}$ to make a first choice. After an initial choice was made, the subject was provided with 1 additional min to make a second choice. All trials ended after the second choice or $1 \mathrm{~min}$ after the first choice, whichever occurred first. The criteria to complete Shaping Phases 4 and 5 were the same as those of the third shaping phase. The birds were required to meet the criteria for 2 consecutive days before they completed Shaping Phase 5 and started testing.

\section{Testing}

Numerous tests were administered to determine which cues controlled the pigeons' choices. Test and control trials were conducted without reinforcement. Control trials were visually identical to training trials but were unreinforced in order to measure behavior in the absence of potential food-related cues. Choices were recorded manually; in addition, test and control trials were video taped. A subject was allotted 5 min to make a choice, after which the trial was terminated.

Geometric Only, No Features, and Identical Features Tests. The first test series given to both groups of birds was designed to assess control by the geometry of the enclosure in the absence of featural information. For the birds in the geometric group, two Geometric Only test sessions, consisting of five baseline trials and five test trials, were given. The environment on test trials was identical to that of baseline trials, but test trials were nonreinforced. The birds in the feature group were administered an Identical Features Test and a No Features Test. Both tests were administered over three sessions with five baseline trials, two control trials, and three test trials per session. The baseline and control trials provided the training environment (i.e., distinctive features in each comer) and were reinforced or nonreinforced respectively. Four identical landmarks or panels (depending on a subject's group designation) replaced the original featural information for the Identical Features Test (see the test landmarks and panels labeled $I$ in Figure 1 for stimulus dimensions), whereas for the No Features Test, all featural information was removed (see Figure 2).

When this testing stage was completed, the feature group moved directly on to the next test series (Distant Features Only Tests), whereas the geometric group subjects were provided with featural information and retrained to choose only one corner. Each subject maintained its positive corner, and the configuration of featural information was identical to that of the feature group. Two birds were assigned to the landmark subgroup and the other 2 were assigned to the panel subgroup. Retraining was conducted in the same manner in which the feature group was initially trained (from Shaping Phase 1 to Shaping Phase 5). After completion of retraining, the geometric group moved on to the Distant Features Only Test.

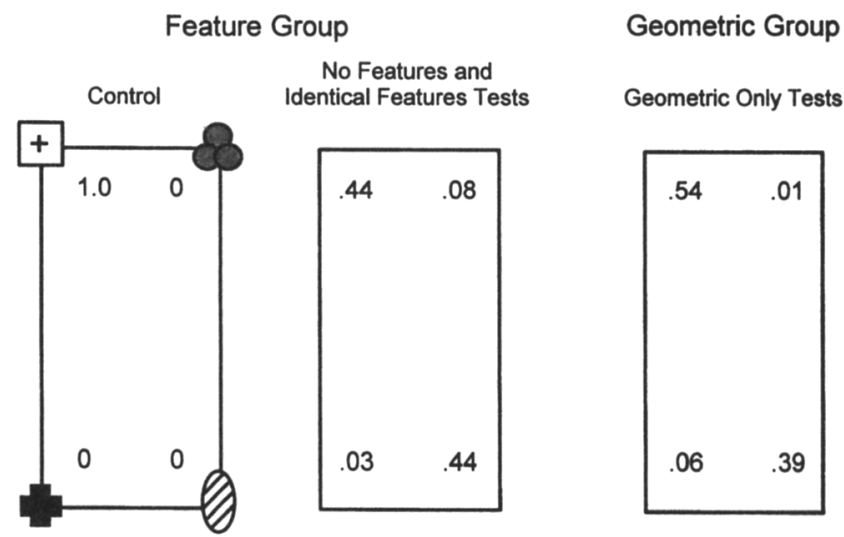

Figure 2. Proportion of choices (rounded to two decimal places) to each corner during Control, No Features, and Identical Features Tests for the feature group and during Geometric Only Tests for the geometric group. Data for the No Features and Identical Features Tests are collapsed, and for all graphs, data are averaged across the 4 birds. Data are shown with the positive comer in the top left; however, the actual positive corner was counterbalanced across birds. Other symbols represent features. 
Distant Features Only Test. This test assessed whether the pigeons had encoded only the features proximal to the goal (the features in the positive corner and its geometric equivalent), or instead had also encoded the featural information present in corners distant from the goal (i.e., in the geometrically incorrect comers). Accordingly, the features in the positive corner and in the geometrically equivalent corner were removed. Thus, only the featural information in the far comers was available for the subjects to distinguish between the positive corner and its geometrical equivalent (see Figure 3). If the subjects relied exclusively on information provided by features near the goal, accuracy would decrease. However, if the birds had encoded the distant features and could use those features to orient themselves, then accurate responses would be maintained despite the removal of the more proximal cues. These tests were conducted over a block of three sessions, with each session consisting of five baseline trials, two control trials, and three test trials.

Transformation tests. The geometric group and the feature group received the same series of transformation tests. These tests were conducted over blocks of three sessions per test. Each testing session consisted of five baseline trials, two control trials, and three test trials. The order in which the transformation tests were administered varied randomly across subjects, with the constraint

\section{Both Groups Combined}
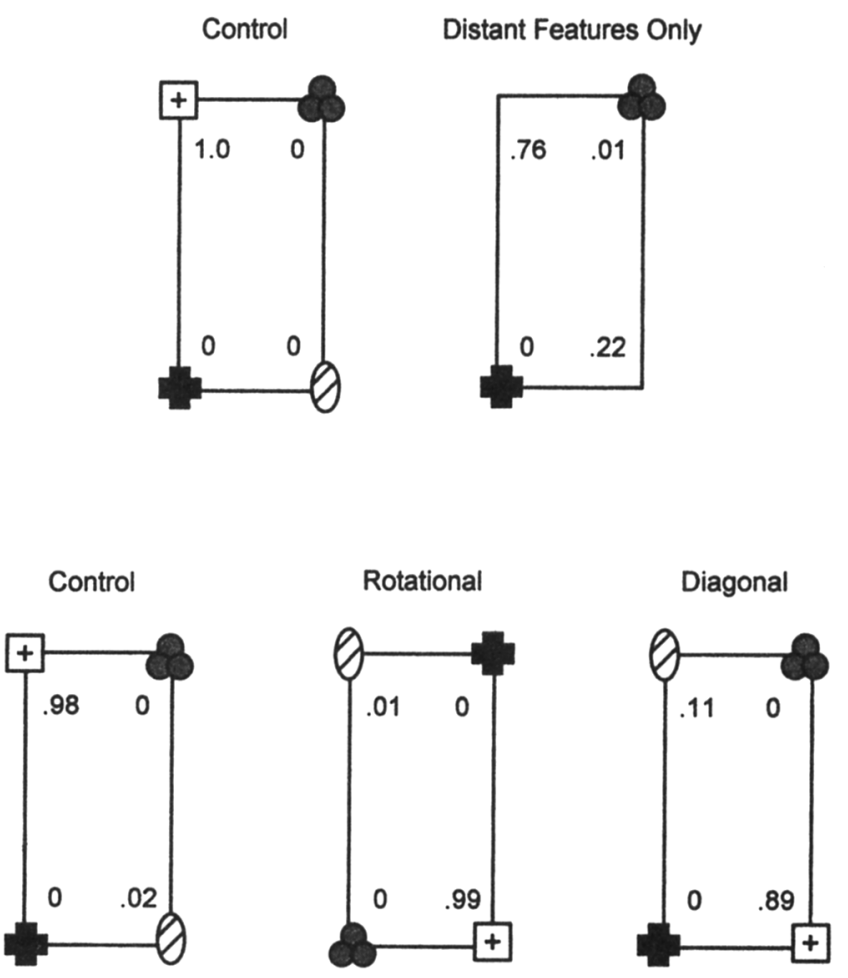

Figure 3. Proportion of choices (rounded to two decimal places) to each corner during Control and Distant Features Only Tests (top row), and during Control, Rotational, and Diagonal Transformation Tests (bottom row). In all graphs, the data are averaged across birds in both groups. The comer containing the positive feature is represented by the square with a plus in the center; however, the actual positive corner was counterbalanced across birds. Other symbols represent features. that each type of transformation test occur in a particular ordinal position for no more than 1 subject in each group.

For two of the transformation tests (Rotational and Diagonal), the relationship between the positive feature and the geometry of the enclosure remained unchanged. The Rotational Transformation Test rotated each feature by two corners (or $180^{\circ}$ ). This test was conducted as a control for unintentional cues that might allow subjects to distinguish between the two geometrically correct corners (e.g., a spot on the wall). If the birds continued to respond to the originally positive corner (now containing a negative feature), this would suggest that the birds' choices were controlled by an unintentional difference between corners. The Diagonal Transformation Test switched the feature at the positive corner with the feature at the geometrically equivalent corner. For the two geometrically correct comers, one corner is correct according to the proximal features, whereas the other corner is correct according to distant features. A schematic of the Rotational and Diagonal Transformation Tests can be seen on the bottom row of Figure 3.

Three tests (the Affine, Reflection, and One Wall Switch Transformation Tests) placed the positive feature and correct geometric corners in conflict (see Figure 4). Therefore, if the subjects relied more on geometry than on featural information, we would expect the majority of responses to be directed to the geometrically correct corners. If the subjects relied primarily upon featural information, the majority of their responses would be directed to the positive feature.

The Affine Transformation altered the environment by rotating the feature in each comer by one position. This test series was presented twice, once with the features rotated by one position clockwise and once with the features rotated by one position counterclockwise.

The Reflection Transformation involved exchanging one pair of features with the features along an opposite wall. This test series was presented twice, once with the features along the long walls switched and once with the features along the short walls switched.

The One Wall Switch Transformation Test involved an exchange of the positive feature with one of the adjacent features. This transformation is different from the Reflection Transformation Test in that the latter switches both pairs of features, whereas the One Wall Switch Transformation Test only involves the exchange of features along one wall. The One Wall Switch Transformation Test was presented twice with one series switching the two features on the long wall and the other switching the two features along the short wall.

Transfer Tests. On completion of the transformation tests, the pigeons were given transfer tests designed to identify which properties of the features had been encoded. The first transfer test administered to all subjects was a Feature Transfer Test in which birds initially trained with the landmarks were tested with the panels (in the same arrangement), and birds initially trained with the panels were tested with the landmarks. The Feature Transfer Test was completed over four sessions, with each session comprising five baseline trials, two control trials, and three test trials. The birds were then given Shape Transfer Tests and Color Transfer Tests, with the order of administration counterbalanced across subjects in each group. A schematic of these tests can be seen in Figure 5. The Color Transfer Test presented the original coloration of the featural information but on novel and identically shaped features (see Figure 1 for an illustration). The type of feature (landmark or panel) was determined by each bird's initial training group. The Shape Transfer Test maintained the original shape of the features, but all the features were of an identical novel color (purple).

New Wall Test. The New Wall Test altered the structure of the experimental apparatus. A new wall was inserted parallel to and $\mathbf{5 0}$ $\mathrm{cm}$ from one of the existing short walls (randomly determined on each trial), thereby forming a new, smaller enclosure with inside dimensions of approximately $50 \times 90 \mathrm{~cm}$. This new wall was 

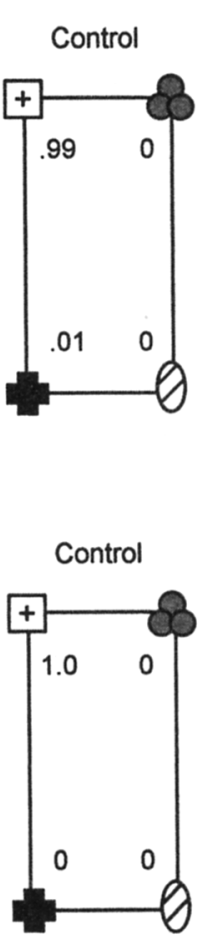

\section{Geometric Group}
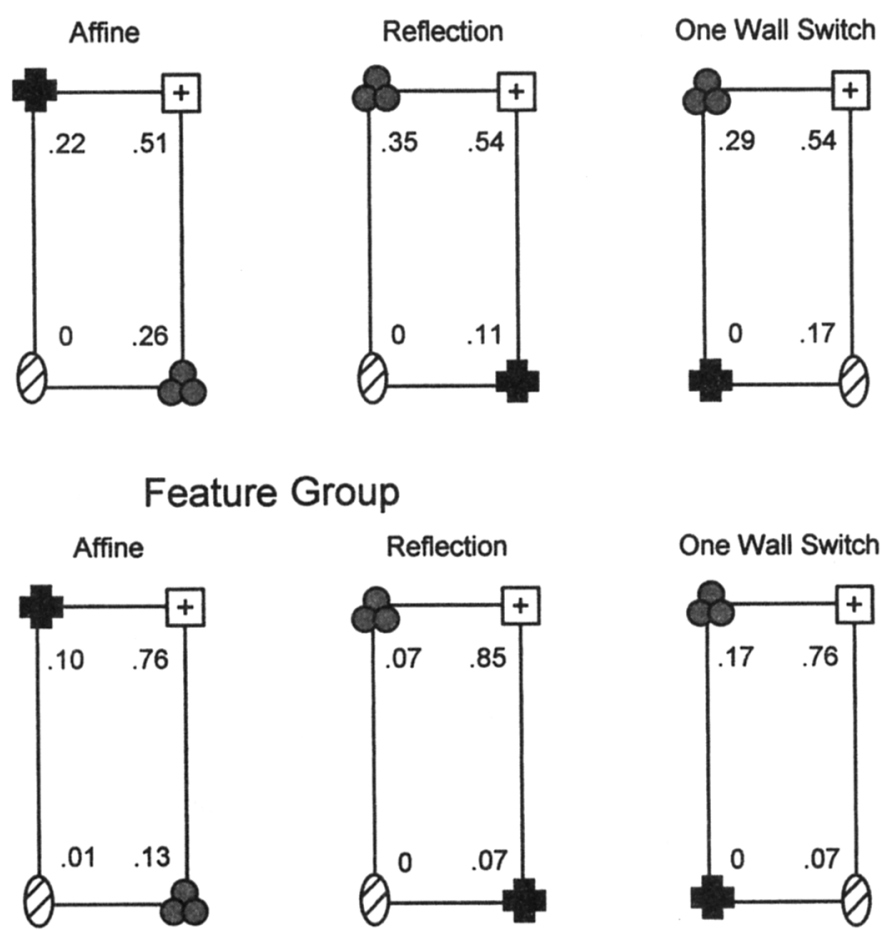

Figure 4. Proportion of choices (rounded to two decimal places) during control tests and during the three transformation tests that pitted features against geometry, averaged across the 4 birds in the geometric group (top row) and the 4 birds in the feature group (bottom row). The corner containing the positive feature is represented by the square with a plus in the center; however, the actual positive corner was counterbalanced across birds. Other symbols represent features.

visually identical to the existing short wall. Two features were moved to the new wall such that the four features maintained the same sense relationship to each other as in training, but the metrics changed because they were now closer together (see Figure 6). Moreover, the new wall changed the relationship between the goal and the geometric shape of the environment. In essence, this manipulation was an affine transformation produced by a contraction along one axis. If the birds' encoding of geometry was based on the encoding of specific distances between objects and walls of the enclosure (i.e., "absolute" geometry), this manipulation would severely distort the geometric information, thereby eliminating geometry as a useful cue. We might expect in such a case that the birds' choices would be controlled exclusively by the positive feature. If, however, the birds encoded a geometric configuration that was invariant to contractions (i.e., relative geometry), the positive feature would now be in a geometrically incorrect corner. We would therefore expect that the binds would show the same pattern of results on this test as they showed on the Affine Transformation Tests.

Geometric Only Test 2. On completion of the New Wall Test, the geometric group was given a second Geometric Only Test. This second Geometric Only Test was identical to the one we gave to this group immediately after shaping.

\section{Data Analysis}

For all statistical tests, the alpha level was set at .05 . The data were analyzed in four steps. First, an analysis was conducted to determine whether the results could be collapsed across tests that were presented twice (first and second Geometric Only Tests) or across tests for which two variations of the same type of test were presented (No Features and Identical Features Tests, clockwise and counterclockwise versions of the Affine Test, long wall and short wall versions of the Reflection Test, and long wall and short wall versions of the One Wall Switch Tests). For this analysis, we used a within-subjects $t$ test on the proportion of correct choices (choices to a geometrically correct corner for the Geometric Only, No Features, and Identical Features Tests, and choices to the featurally correct corner for the Affine, Reflection, and One Wall Switch Tests). In each case, the proportion scores were first subjected to an arcsin transformation (Winer, 1971) to normalize the variance. Because none of these $t$ tests revealed a significant difference, the data were collapsed in each of these cases.

Second, each set of test results was analyzed for differences between the feature and geometric groups. For this analysis, we used between-subjects contrasts with dichotomous categories (Marascuilo \& Serlin, 1988). In each case, choices were divided into two categories. For the tests assessing control by geometry only (i.e., the Geometric Only, the Identical Features, and the No Features Tests), the data were divided into choices of a geometrically correct corner and other choices. For all other tests, the data were divided into choices of a featurally positive corner and other choices. Dichotomous categories contrasts were preferable to a $t$ test because these contrasts are sensitive to the frequency of observations made for each subject. In cases for which the contrast did not 
reveal a significant difference between groups, the data were collapsed across groups for the third analysis.

In the third analysis, we used single-sample $t$ tests to determine whether the proportion of choices made to a particular corner or pair of corners was significantly above chance. For each analysis, the proportion scores were again subjected to an arcsin transformation. The specific proportions analyzed depended on the type of test, as described below in the Results section.

In a final analysis, we collapsed across the two groups and then conducted contrasts (again using the dichotomous categories method) to determine whether birds trained with landmarks responded differently than birds trained with panels during any of the tests. For the Geometric Only Tests, the data were divided into choices of a geometrically correct corner and other choices. For all other tests, the data were divided into choices of a featurally positive corner and other choices.

\section{Results}

The birds in the feature group completed shaping in a mean of 8.75 sessions. Birds in the geometric group completed shaping without features in a mean of 10.25 sessions. This difference was not significant, $t(6)=1.68$.

\section{Both Groups Combined}
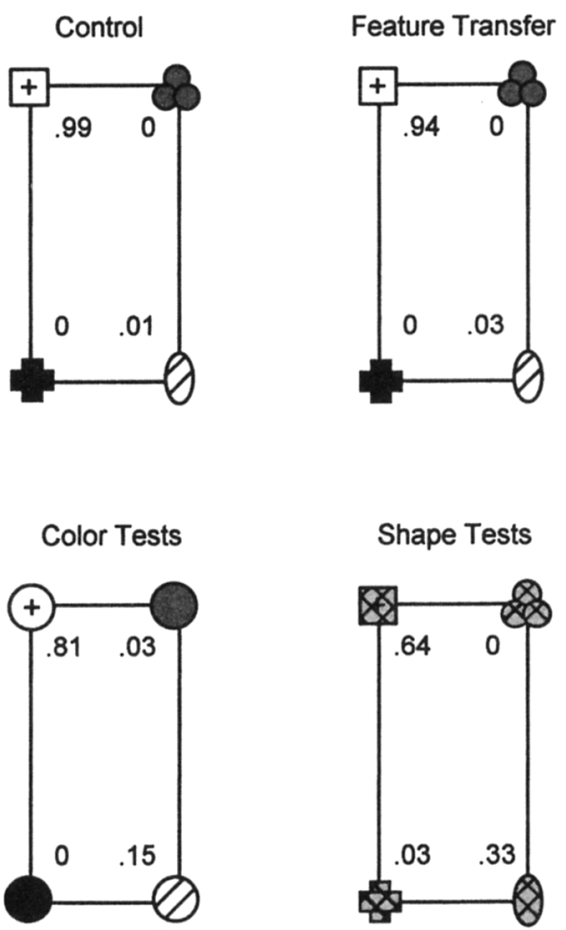

Shape Tests

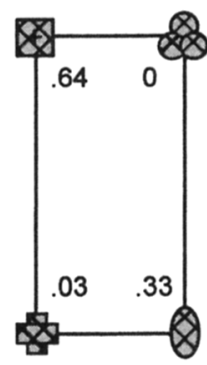

Figure 5. Proportion of choices (rounded to two decimal places) to each corner during Control and Feature Transfer Tests (top row), and during Color and Shape Tests (bottom row). In all graphs, the data are averaged across birds in both groups. The corner containing the positive feature is shown in the top left; however, the actual positive corner was counterbalanced across birds. Other symbols represent features.
Both Groups Combined
Control

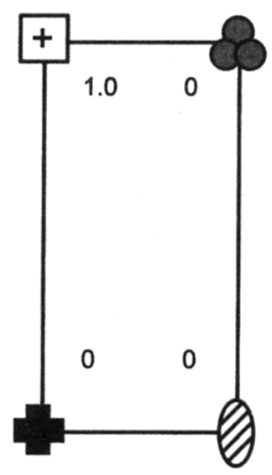

New Wall

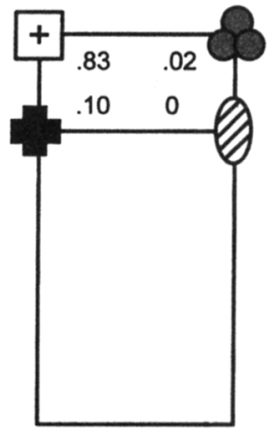

Figure 6. Proportion of choices (rounded to two decimal places) to each corner during Control and New Wall Tests, averaged across birds in both groups. The corner containing the positive feature is shown in the top left; however, the actual positive corner was counterbalanced across birds. Other symbols represent features.

When the birds in the geometric group were subsequently trained with features, they completed shaping in a mean of 12 sessions (with 3 birds taking 8 sessions and 1 bird taking 24 sessions), which was again not significantly different than the sessions required by the feature group, $t(6)=0.81$. Collapsing across the two groups, the number of sessions required to complete shaping with features was not significantly different for birds trained with landmarks $(M=12)$ than for birds trained with panels $(M=8.75), t(6)=0.81$.

\section{Identical Features, No Features, and Geometric Only Tests}

As can be seen in Figure 2, regardless of initial training, the pigeons did not respond randomly when placed within the environment void of relevant featural information; instead, they chose a geometrically correct corner most of the time. The Identical Features and No Features Test results for the feature group and the Geometric Only Test results for the geometric group were collapsed and analyzed together because the contrast revealed no statistically significant difference between the groups, $z=0.80$. To assess the reliability of preferences for a geometrically correct corner, we analyzed the proportion of total choices that were made to a geometrically correct corner with a one-sample $t$ test, which revealed that the subjects chose geometrically correct corners more often than expected by chance $(0.50), t(7)=$ 19.49. We further analyzed choices of a geometrically correct corner according to whether they were made to the positive corner (i.e., the one that contained food during training) or its geometrical equivalent. The mean proportion of responses to the positive corner was 0.544 , which was not significantly above $0.50, t(7)=1.09$. Thus, as expected, the birds distributed their responses approximately equally between these two geometrically correct corners. 


\section{Distant Features Only Tests}

Results of the Distant Features Only Tests are shown in the top row of Figure 3. The two groups of subjects did not show a statistically significant difference in their proportion of choices to the correct corner, $z=0$; therefore, we collapsed their results. The Distant Features Only Test assessed whether the pigeons were able to locate their positive corner when the features at the positive corner and the geometrically equivalent corner were removed. If subjects showed no control by the distant cues, we would expect them to respond in the same way as they did on Geometric Only Tests and hence we would expect them to choose randomly between the two geometrically correct corners. In a first analysis, we confirmed that the pigeons chose one of the two geometrically correct corners more often than expected by chance $(0.50), t(7)=42.5$. To determine whether the pigeons distinguished between the two geometrically correct corners, we next analyzed choices of the positive corner as a proportion of total choices made to either of the two geometrically correct corners. A onesample $t$ test revealed that the proportion of choices to the positive corner was significantly above chance $(0.50), t(7)=$ 6.01 . Therefore, the birds did not respond randomly between the two geometrically correct corners, indicating that they were able to use the distant features to orient themselves.

\section{Transformation Tests With Correct Geometry and Positive Feature}

Results of the two transformation tests that maintained the relationship between the positive feature and the geometric shape of the environment (Rotational and Diagonal) are shown in the bottom row of Figure 3 . We found no statistically significant difference between groups on either the Rotational Test, $z=1.06$, or the Diagonal Test, $z=1.60$; hence, we collapsed the results across groups for each of these tests. For both of these transformations, the positive feature was moved to a new geometrically correct corner. In a first analysis, we confirmed that the pigeons chose one of the two geometrically correct corners more often than expected by chance $(0.50), t(7)=42.5$. To determine whether the pigeons distinguished between the two geometrically correct corners, we next analyzed choices of the corner containing the positive feature as a proportion of total choices made to either of the two geometrically correct corners. One-sample $t$ tests revealed a statistically significant preference (i.e., greater than 0.50 ) for the corner containing the positive feature for both the Rotational Test, $t(7)=42.46$ and for the Diagonal Test, $t(7)=10.41$.

\section{Transformation Tests With Geometry and Features in Conflict}

For each of the transformation tests that pitted geometry against features (the Affine, Reflection, and One Wall Switch Transformation Tests), the contrasts showed a significant difference between the two groups (Affine, $z=3.38$; Reflection, $z=5.36$; One Wall Switch, $z=4.00$ ), with the feature group choosing the corner containing the positive feature more often than the geometric group. Results for each group are shown in Figure 4.

For each of these tests, there was one featurally correct corner, two geometrically correct corners, and one corner that was incorrect on the basis of both geometry and features. For both groups and in each of the three tests, choice of the corner that was incorrect on the basis of both features and geometry either never occurred or occurred significantly less often than expected by chance $(0.25)$, $t(3)=79.02$ (the feature group during the Affine Tests). Clearly then, the birds did not choose randomly but instead chose on the basis of either the featural or the geometric information or both. To determine whether the birds in each group showed a significant preference for featural information over geometric information, we next analyzed choices to the featurally correct corner as a proportion of total choices made to either the featurally correct or the geometrically correct corners. A random choice among the three corners that were correct according to either geometry or features would yield a chance level proportion of 0.33 . The feature group chose the featurally positive corner significantly more often than expected by chance during the Affine Transformation, $t(3)=7.64$, and the Reflection Transformation, $t(3)=8.80$, but not during the One Wall Switch Transformation, $t(3)=2.51$. The geometric group did not choose the featurally correct corner significantly more than chance on any of the three transformations, $t(3)=2.89$, $t(3)=0.64$, and $t(3)=0.71$, respectively.

\section{Transfer Tests}

The two groups did not differ significantly on any of the transfer tests (Feature Transfer, $z=0.78$; Color Transfer, $z=0.10$; Shape transfer, $z=1.62$ ); therefore, we combined the results for the two groups. Results of these tests are shown in Figure 5. For the Feature Transfer, Color Transfer, and Shape Transfer Tests, the birds chose one of the two geometrically correct corners significantly more often than expected by chance $(0.50), t(7)=25.64,25.64$, and 37.98 , respectively. To determine whether the birds were able to use the manipulated featural information to distinguish between the two geometrically correct corners, we next analyzed choices to the positive corner as a proportion of total choices made to either of the two geometrically correct corners for each test and compared these proportions to the chance level of 0.50 . During the Feature Transfer Tests, choice of the featurally correct corner was greater than chance, $t(7)=$ 5.26 , demonstrating that the pigeons transferred accurately between the landmarks and panels or vice versa. During the Color Transfer Tests, providing the subjects with the correct color while altering the shape of the features did not eliminate accurate choice; subjects chose the corner containing the feature with the positive color significantly more often than expected by chance, $t(7)=6.67$. However, accurate choice was not maintained during the Shape Transfer Tests in which the color of the features was altered while maintaining the original shape; the subjects did not 
choose the feature of positive shape significantly more often than chance, $t(7)=2.07$.

\section{New Wall Test}

Results of the New Wall Test are shown in Figure 6. No statistically significant difference was found between the two groups, $z=1.55$; therefore, we collapsed the data. On this test, there was one featurally correct corner, two corners that were correct according to relative geometry (none of the corners were correct according to absolute geometry), and one corner that was incorrect according to both geometry and features. Choice of the corner that was incorrect on the basis of both sources of information never occurred, indicating that at least one of these two sources of information controlled the birds' choices. To determine whether the birds showed a significant preference for the corner with the correct feature, we assessed their choices of the featurally correct corner as a proportion of choices of the featurally correct corner plus choices of the two corners that were correct according to relative geometry. The one-sample $t$ test showed that choice of the featurally correct corner was significantly above chance $(0.33), t(7)=5.60$.

\section{Comparisons Between Landmark-Trained and Panel-Trained Subjects}

Comparisons between birds trained with landmarks and birds trained with panels did not reveal systematic differences. Although contrasts between the birds trained with landmarks versus the birds trained with panels revealed significant differences in 4 of the 11 tests, the direction of the difference varied. Choice of the featurally correct corner was significantly higher for the birds trained with landmarks than for the birds trained with panels in the One Wall Switch Test, $z=4.91$; the Shape Transfer Test, $z=3.26$; and the New Wall Test, $z=3.30$. However, during the Affine Test, choice of the featurally correct corner was significantly higher for the birds trained with panels than for the birds trained with landmarks, $z=2.25$.

\section{Discussion}

The pigeons in this experiment learned to locate hidden food on the basis of information contained within a rectangular apparatus. This experiment differed from previous studies investigating encoding of features and geometry in that two groups of birds were initially trained with either the featural information present or absent. We found the initial training received by the pigeons to be an important determinant of how the environmental information controlled choice behavior.

The Identical Features, No Features, and Geometric Only Tests examined whether both groups encoded the geometric information supplied by the environment regardless of whether it was required to solve the task. Pavlovian conditioning mechanisms exhibit overshadowing of a cue by other, more salient cues with equal predictive validity (e.g., Kamin, 1969). Although overshadowing has been shown in the spatial domain (March, Chamizo, \& Mackintosh, 1992; Spetch, 1995), there was no evidence that it occurred here. In this experiment, training pigeons to locate the goal in the presence of featural information did not interfere with their subsequent ability to find the goal on the basis of geometric information only.

The Distant Features Only Test investigated the content of featural information being encoded: Did pigeons encode just the feature in the positive corner or some larger portion of the array (e.g., the features in the distant corners)? Both groups demonstrated the ability to use the distant features to correctly locate the positive comer. This result contrasts with previous studies that found that neither rats (Cheng, 1986) nor chicks (Vallortigara et al., 1990) showed evidence of encoding the distant features within a geometric enclosure. A difference in encoding between rats and pigeons is perhaps not surprising given that these two species belong to different classes of animals and inhabit contrasting ecological niches. The present study, however, suggests a difference between two avian species in the encoding of cues distant to the goal; distant cues were used by the pigeons in our study but not by the chicks in the study by Vallortigara et al. These contrasting results between pigeons and chicks could reflect any of a number of differences between the studies, including differences in the procedures, the nature of the search response (pecking versus scratching), or the specific apparatus and featural cues used. Another interesting possibility is that developmental factors (either maturation or experience) may affect encoding. In support of this possibility, developmental factors have been implicated in the encoding of featural information by humans (Hermer \& Spelke, 1994). An interesting area for future research would be to directly investigate whether use of featural information for navigation emerges or broadens during development in an avian species.

During the transformation tests, the two groups of pigeons responded to alterations in the featural configuration in a systematically different way. Transformation tests that maintained the relationship between the correct feature and the geometry of the environment (Diagonal and Rotation Transformation) did not reveal a difference in responding between the two groups. However, if the positive feature was placed in a geometrically incorrect position (Affine, Reflection, and One Wall Switch Transformation Tests) the distribution of responses made by the two groups was systematically different. When presented with conflicting information, the birds in the geometric group shared their responses between the geometrically correct corners and the featurally correct corner. In contrast, the feature group demonstrated primary stimulus control by the feature itself. Thus, initial training with geometric information as the single reliable cue seems to have reduced the relative control by featural information. Features did exert some control in the geometric group, however. It seems reasonable to attribute this control to the necessity of using featural information to discriminate which of the two geometrically correct corners was the goal.

The transfer tests were conducted to investigate which properties of the features exerted the most stimulus control. These tests revealed that pigeons were able to transfer 
accurately between two-dimensional panels and threedimensional landmarks and vice versa. This transfer, together with the lack of consistent differences between birds trained with landmarks and birds trained with panels, appears to contrast with results by Chappell and Guilford (1997). They found that pigeons were unable to accurately locate a goal using two-dimensional cues affixed to the walls of an enclosure but that they were able to locate the goal using a three-dimensional landmark placed on the floor of the enclosure. However, their panels were located on walls on either side of the goal rather than directly behind the goal. An interesting future experiment would be to directly compare control by panels on the side walls (as in Chappel and Guilford) with control by a panel placed in the comer behind the goal (as in our experiment).

Separation of the two dominant properties of the features, color and shape, revealed color to be encoded predominately for both groups of subjects. It is interesting that although the groups differed in the encoding of feature versus geometry, both groups encoded the same dimension of featural information. The finding that color was the dominant featural property encoded is consistent with the finding by Spetch and Mondloch (1993) that pigeons' spatial search in a touch-screen task was controlled more by the color of a landmark than by the shape of a landmark.

The New Wall Test investigated a very interesting aspect of the metric frame. This test showed that when the dimensions of the environment were altered quite radically, both groups of birds relied on the positive feature. The responses of utmost interest are those by the geometric group. The New Wall Test was essentially an Affine with a contraction of the search space: The positive feature was located at a geometrically incorrect comer according to relative geometry (i.e., geometry that is invariant to contraction). Therefore, if pigeons were sensitive to relative geometry, they should respond in the same way to this test as they did to the Affine Transformation Tests. During Affine Tests, birds in the geometric group showed partial control by the environmental geometry, and, in contrast to the birds in the feature group, they did not show a significant preference for the corner containing the positive feature. During the New Wall Test, however, birds in the geometric group responded in the same way as did birds in the feature group: They showed primary control by the feature. This finding suggests that the geometric information was probably encoded according to absolute metrics rather than relative geometry. If the birds encoded the geometry in terms of absolute metrics (e.g., the absolute lengths of the walls on either side of the positive corner), the New Wall manipulation would distort the environment to the point that none of the corners would be geometrically correct (i.e., there would no longer be a 190 -cm wall). In such a case, the birds in both groups would be expected to use featural cues, which is consistent with the results we obtained. In retrospect, an interesting manipulation would have been to combine the New Wall Test with removal of all featural information from the environment. If encoding of geometry is indeed absolute, the distribution of responses would have been random during such a test.
Our suggestion that the pigeons probably encoded the geometric information in terms of absolute rather than relative metrics seems consistent with results of recent experiments in which pigeons were trained to find a goal in the center of a landmark array and then were tested with expansions of the landmark array (Spetch, Cheng, \& MacDonald, 1996; Spetch et al., 1997). In tasks conducted both on the monitor screen and on the laboratory floor, pigeons responded to the expansions of the landmark array by maintaining the correct absolute distance from individual landmarks rather than by responding to the correct relative location in the array (i.e., they rarely searched at the center of the expanded array). Interestingly, a recent study conducted with young chickens (Tommasi, Vallortigara, \& Zanforlin, 1997) obtained somewhat different results. The chickens were trained to find food hidden in the center of an enclosure and then the size or shape of the enclosure was varied on tests. Although the chickens sometimes searched at the absolute training distance from the walls of the enclosure, they more often searched at the center of new enclosures. Determining the nature of these contrasting results for pigeons and young chickens is an interesting topic for future research.

In summary, our results suggest two main conclusions. First, we have shown that pigeons, similar to rats, chicks, and young children, encode the geometric shape of an environment, even when the presence of featural cues makes encoding of geometry unnecessary. Cheng's (1986) research suggested that rats primarily use a purely geometric module for navigation. Although rats could use features to distinguish the correct corner from its geometric equivalent, they did not follow the correct feature when it was placed in a geometrically incorrect corner. Consequently, Cheng suggested that rats do not encode features independently of the geometry, but instead "paste the requisite features onto the requisite frame" (p. 176). In contrast, chicks in the study by Vallortigara et al. (1990) and pigeons in the feature group of our study appeared to encode features independently of geometry because they followed the correct feature when it was placed in a geometrically incorrect corner. These comparisons suggest that encoding of geometric information may be quite general, but the primacy of geometric information appears to vary across species.

Second, our research revealed that the primacy of control by geometric and featural cues is determined not only by species' dispositions but also by experiential factors. Pigeons that were trained without features before being trained with features responded to tests that pit geometry against featural information in a manner similar to that of rats and different from that of pigeons trained with features from the outset. That is, pigeons in the geometric group did not show a significant level of choice of the positive feature when it was moved to a geometrically incorrect corner. Instead, they distributed their responses among the corner containing the positive feature and the two geometrically correct corners. Thus, at least for pigeons, initial experiences can alter the primacy of control by geometric and featural information. 


\section{References}

Brodbeck, D. R. (1994). Memory for spatial and local cues: A comparison of a storing and a nonstoring species. Animal Learning and Behavior, 22, 119-133.

Chappell, J., \& Guilford, T. (1997). The orientational salience of visual cues to the homing pigeon. Animal Behaviour, 53, 287-296.

Cheng, K. (1986). A purely geometric module in the rat's spatial representation. Cognition, 23, 149-178.

Cheng, K., \& Gallistel, C. R. (1984). Testing the geometric power of an animal's spatial representation. In H. L. Roitblat, T. G. Bever, \& H. S. Terrace (Eds.), Animal cognition (pp. 409-423). Hillsdale, NJ: Erlbaum.

Cheng, K., \& Spetch, M. L. (1998). Mechanisms of landmark use in mammals and birds. In S. Healy (Ed.), Spatial representation in animals (pp. 1-17). Oxford: Oxford University Press.

Gallistel, C. K. (1990). The organization of learning. Cambridge, MA: MIT Press.

Hermer, L., \& Spelke, E. (1994). A geometric process for spatial representation in young children. Nature, 370, 57-59.

Hermer, L., \& Spelke, E. (1996). Modularity and development: The case of spatial reorientation. Cognition, 61, 195-232.

Kamin, L. J. (1969). Selective association and conditioning. In N. J. Mackintosh \& W. K. Honig (Eds.), Fundamental issues in associative learning (pp. 42-64). Halifax, Nova Scotia, Canada: Dalhousie University Press.

Marascuilo, L. A., \& Serlin, R. C. (1988). Statistical methods for the social and behavioral sciences. New York: W.H. Freedman.

March, J., Chamizo, V. D., \& Mackintosh, N.J. (1992). Reciprocal overshadowing between intra-maze and extra-maze cues. Quarterly Journal of Experimental Psychology, 45B, 49-63.

Margules, J., \& Gallistel, C. R. (1988). Heading in the rat: Determination by environmental shape. Animal Learning and Behavior, 16, 404-410.

Poucet, B. (1993). Spatial cognitive maps in animals: New hypotheses on their structure and neural mechanisms. Psychological Review, 100, 163-182.
Spetch, M. L. (1995). Overshadowing in landmark learning: Touch-screen studies with pigeons and humans. Journal of Experimental Psychology: Animal Behavior Processes, 21, 166181.

Spetch, M. L., Cheng, K., \& MacDonald, S. E. (1996). Learning the configuration of a landmark array: I. Touch-screen studies with pigeons and humans. Journal of Comparative Psychology, 110. 55-68.

Spetch, M. L., Cheng, K., MacDonald, S. E., Linkenhoker, B. A., Kelly, D. M., \& Doerkson, S. R. (1997). Use of landmark configuration in pigeons and humans: II. Generality across search tasks. Journal of Comparative Psychology, 111, 14-24.

Spetch, M. L., \& Edwards, C. A. (1988). Pigeons', Columba livia, use of global and local cues for spatial memory. Animal Behaviour, 36, 293-296.

Spetch, M. L., \& Mondloch, M. V. (1993). Control of pigeons' spatial search by graphic landmarks in a touch-screen task. Journal of Experimental Psychology: Animal Behavior Processes, 19, 353-372.

Strasser, R., \& Bingman, V. P. (1996). The relative importance of location and feature cues for homing pigeon (Columba livia) goal recognition. Journal of Comparative Psychology, 110, 77-87.

Tommasi, L., Vallortigara, G., \& Zanforlin, M. (1997). Young chickens learn to localize the center of a spatial environment. Journal of Comparative Physiology A-Sensory Neural and Behavioral Physiology, 180, 567-572.

Vallortigara, G., Zanforlin, M., \& Pasti, G. (1990). Geometric modules in animals' spatial representations: A test with chicks (Gallus gallus domesticus). Journal of Comparative Psychology, 104, 248-254.

Winer, B. J. (1971). Statistical principles in experimental design (2nd ed.). New York: McGraw-Hill.

Received July 30, 1997

Revision received January 16, 1998 Accepted January 16, 1998 